\title{
Lettres et documents comptables inédits sur Mgr Albert Bailly et sur les eaux minérales de Courmayeur
}

\section{Gianni Mombello}

\section{(2) OpenEdition Journals}

\section{Édition électronique}

URL : http://journals.openedition.org/studifrancesi/34136

DOI : 10.4000/studifrancesi.34136

ISSN : 2421-5856

Éditeur

Rosenberg \& Sellier

\section{Édition imprimée}

Date de publication : 1 novembre 2005

Pagination : $357-376$

ISSN : 0039-2944

\section{Référence électronique}

Gianni Mombello, «Lettres et documents comptables inédits sur Mgr Albert Bailly et sur les eaux minérales de Courmayeur », Studi Francesi [En ligne], 146 (XLIX | II) | 2005, mis en ligne le 30 novembre 2015, consulté le 19 avril 2021. URL : http://journals.openedition.org/studifrancesi/34136 ; DOI : https://doi.org/10.4000/studifrancesi.34136

\section{(c)}

Studi Francesi è distribuita con Licenza Creative Commons Attribuzione - Non commerciale - Non opere derivate 4.0 Internazionale. 


\section{Lettres et documents comptables inédits sur Mgr Albert Bailly et sur les eaux minérales de Courmayeur}

Les services rendus par le Savoyard Antoine-Philibert Bailly à ses seigneurs naturels sont mieux connus depuis que l'importante correspondance qu'il a envoyée à la cour de Turin et qui est conservée aux Archives de l'Etat de cette ville a commencé à être publiéé.

Dès avant son entrée dans la congrégation des Clercs réguliers de Saint Paul $(\text { Barnabites })^{2}$, où il prit le nom d'Albert, il avait reçu cinquante ducatons de la part de Charles-Emmanuel I, le 27 janvier 1629 (document I) ${ }^{3}$. Cette gratification devait se rapporter aux services que le jeune Bailly avait rendus, au cours de la première guerre de Gênes, commencée en avril 1625 et qui se solda par un échec ${ }^{4}$, ainsi qu'à d'autres dont la nature est difficile à préciser. En 1672, au moment de la seconde guerre de Gênes, Mgr Bailly rappelait dans un mémoire ${ }^{5}$ la part qu'il avait prise, en qualité de secrétaire, au cours de la première guerre contre cette république.

D'après un autre document du 27 janvier 1632, signé par le père Angelo Roncallio (Roncaglia) ${ }^{6}$, il ressort que Bailly avait passé sept ans au Piémont, donc à partir d'environ la moitié de l'année 1624 à la moitié de l'année 1631. Dès l'automne 1630 au printemps 1631, il avait accompagné la duchesse Marie-Christine de France, fuyant Turin et la peste, à Cherasco où fut signé le 16 avril 1531, le traité qui porte le nom de cette ville. Durant ces mois, il a joué le rôle de secrétaire, comme il le rappelait dans sa vieillesse'. Avant cela il avait suivi des cours de droit à l'Université de Turin, durant une année et demie, pris les quatre ordres mineurs et posé sa candidature pour être accueilli chez les Barnabites auprès du couvent de San Dalmazzo sis dans la même ville.

Au moment où il aurait dû prononcer sa profession solennelle, le père Joseph de Sales découvrit que ce postulant avait laissé des dettes à Turin, c'est pourquoi on le

(1) Après un premier volume paru en 1992 [L. Giachino, Lettres inédites de Mgr Albert Bailly (Rome - 1658), Aoste, Imprimerie Valdôtaine, 1992, «Bibliothèque de l'Archivum Augustanum, vol. XXVI], six autres ont été publiés à Aoste dans les volumes $1,2,3,4,6$ et 7 de la série «Ecrits d'histoire, de littérature et d'art» de l'Académie SaintAnselme: La correspondance d'Albert Bally, publiée sous la direction de Gianni Mombello. Volume I, années 1643-1648 par Luca Giachino (1999); vol. II, années 1649-1650, par Paola Cifarelli (1999); vol. III, année 1651, par Antonella Amatuzzi (1999); vol. IV, années 1652-1653 par Giorgia Puttero (2001); vol. V, années 1654-1655, par Paola Cifarelli (2003); vol. VI, années 1656-1658, par Laura Ghiosso (2004); vol. VII, années 1659-1663, par Giorgia Puttero (2005). Les trois derniers volumes sont en cours de rédaction par Stefania Vignali, Antonella Amatuzzi et Giorgia Puttero.

(2) G. Mombello, La jeunesse d'Antoine-Philibert (Albert) Bailly d'après des documents inédits, in Albert Bailly évêque d'Aoste, trois siècles après (1691-1991). Actes du Colloque international d'Aoste (11-12 octobre 1991) réunis par M. Costa,
Aoste, Imprimerie Valdôtaine 1993 [«Bulletin de l'Académie Saint-Anselme», nouvelle série, vol. IV], pp. 25-65.

(3) Ibid., p. 34

(4) G. Amoretti, Il ducato di Savoia dal 1559 al 1713, Torino, Daniela Piazza Editore, 1984-1988, en 4 voll. t. II, dal 1610 al 1659, pp. 27-30.

(5) Archivio di Stato di Torino (abrégé: AST), Lettere vescovi. Aosta, 1 , fasc. 7 , pièce 62 , f. $1 \mathrm{r}$.-v. Texte reproduit en partie dans $\mathrm{G}$. Mombello, $L a$ jeunesse d'Antoine-Philibert (Albert) Bailly d'après des documents inédits, éd. cit., pp. 32-33.

(6) G. Mombello, La jeunesse d'Antoine-Philibert (Albert) Bailly d'après des documents inédits, éd. cit., pp. 54-55. Ce document est conservé à Milan dans l'Archivio Storico di San Barnaba, cart. H-XXI, liasse: De recipiendis in congregatione, fasc. 76.

(7) AST, Lettere vescovi. Aosta, liasse 2, fasc. 1, lettre 148 du 26 juillet 1680: «J'ay eté secretaire d'une grande princesse (Marie-Christine de France), comme Mr. le president de Lescheraine l'est au-jourd'-huy de V.A.R. (Marie-Jeanne-Baptiste)». Cfr. aussi document I. 
renvoya dans cette ville, accompagné du père Juste Guérin, futur évêque de Genève, parce que Bailly pensait qu'il était pour lui «cosa facile l'ottener somma sofficiente per sodisfare a' suoi creditori, per mezzo dell'Altezze Serenissime alle quali egli ha servito di secretaro (document $\mathrm{I})^{8}$ et pretende anche essergli là dovuto qualche cosa» ${ }^{9}$.

Cette mission a dû avoir du succès puisque Dom Albert Bailly fut admis à la profession solennelle qu'il prononça le 2 octobre $1633^{10}$. En effet, le 20 août de cette année-là, le duc Victor-Amédée II lui octroyait trois cents ducatons «per impiegarli in un oppera pia». C'est notre document II. Cette somme, qui aurait pu être prélevée aussi par «un suo legitimo agente», a donc été jugée suffisante pour satisfaire ses créditeurs.

On peut suivre pas à pas les événements qui touchent à la vie de ce Barnabite grâce aux Registri delle lettere dei Prepositi Generali qu'un de mes anciens étudiants a transcrites dans son mémoire de mâitrise ${ }^{11}$.

Envoyé au collège de Lescar, il reçut le diaconat, le 2 juin $1635^{12}$ et ensuite la prêtrise ${ }^{13}$ des mains de Mgr Jean-Henry de Salette. Au cours de son séjour en Béarn, il déploya une intense activité de controversiste contre les Protestants ${ }^{14}$ et surtout il témoigna son habilité «nel maneggio temporale», en sa qualité de vicaire de cette maison ${ }^{15}$, c'est pourquoi, dès mai 1641 il fut envoyé à Paris pour suivre de près un problème de bénéfices, dont la solution avait été transférée du Parlement de Toulouse au Conseil du roi ${ }^{16}$. C'est à Paris que le Père Albert devait séjourner, quoique avec de courtes interruptions, jusqu'au début de $1658^{17}$, d'abord comme supérieur de

(8) Cfr. aussi la note 7 précédente.

(9) G. Mombello, La jeunesse d'Antoine-Philibert (Albert) Bailly d'après des documents inédits, éd. cit., p. 61. Lettre du 22 février 1633 signée par Dom Lazzaro Roncaglio. Ce document est conservé dans les mêmes archives des Barnabites de Milan, cote: cart K-X: De admittendis ad professionem in Congregatione, liasse 2, doc. 9.

(10) Ibid., pp. 63-64. Document conservé à Milan, dans les mêmes archives, classé: cart. L-IV: Formulae professionis a clericis emissa, liasse unique, n. 708. Selon le père Sergio Pagano [Gerarchia barnabitica. Prepositi generali - Assistenti generali - Procuratori generali - Prepositi provinciali - Prepositi e superiori locali, vol. I (1536-1700), Roma, Centro Studi Padri Barnabiti, 1994 («Subsidia studiorum», 1)], p. 165, n. 1099, Dom Bailly aurait fait sa profession le 30 novembre 1633 .

(11) Il s'agit du lieutenant Olindo Pagliaro. Pour la rédaction de son mémoire présenté au «Corso di Laurea Interfacoltà in Scienze Strategiche», au cours de l'année académique 2002-2003 et intitulé: Contributo allo studio della corrispondenza di Mgr. Albert Bailly il a grandement profité de la compétente et aimable aide des Révérends Pères Giuseppe Maria Cagni et Mauro Regazzoni qui sont les responsables de l'Archivio Storico dei Barnabiti - Roma, SS Biagio e Carlo a' Catinari. Qu'ils veuillent trouver ici l'expression de sa vive gratitude et de la mienne. Cette institution sera citée dorénavant sous le sigle ASBR. Le sigle RLPG renvoie aux Registri delle lettere dei Prepositi Generali. Prima serie, et le mémoire de M. Olindo Pagliaro sera cité par son seul nom suivi du numéro de la page. Le sigle E, b renvoie au Liber tertius professionum de 1605 à 1671 .

En ce qui concerne les dettes de Dom Bailly et son allée à Turin pour s'en acquitter: cfr. ASBR -RLPG, I ire série, vol. 32, ff. 219-20, 223-24, 356, 449-50. Le résumé des lettres du supérieur général aux pères de Thonon a été transcrit par PAGLIARO, pp. 175-78.

(12) ASBR, E. b, Liber tertius professionum clericorum. A die 7 septembris anni 1605 ad diem 23 augusti 1671, 827, p. 156. PAGLIARO, p. 252.

(13) ASBR-RLPG, I ire série, vol. 34, f. 537 et vol. 35, f. 9 ( lettres au père prévôt Lazzaro Roncaglia du 16 juin 1635: «Giudicando idoneo al sacerdozio D. Alberto, lo promuovi sanza altra patente», lettre du 15 juillet au même père: «mandi quanto prima $D$. Alberto al Sacerdozio»). E. b (Liber tertius professionum clericorum a die 7 septembris anni 1605 ad diem 23 augusti 1671), p. 156. La date de son ordination sacerdotale est laissée en blanc. PAGLIARO, pp. 179, 181 et 252. Selon le père S. PAGANO, Gerarchia barnabitica. Prepositi generali-Assistenti generali-Procuratori generali - Prepositi provinciali - Prepositi e superiori locali, éd. cit., vol. I, p. 165, n. 1099, son ordination sacerdotale remonte à 1636 ,

(14) De cette activité il nous reste une Lettre du reverend pere Dom Albert Bally [...] a Monseigneur l'illustrissime et reverendissime Jean-Henry de Salette evesque de Lescar, A Lescar, par Pierre Compagne, 1640 (exemplaire à Paris, BnF. D 5980) au sujet d'une controverse avec le sieur Abbadie ministre à Pau. De ses Disputationes de traditionibus apostolicis contra haereticos, Pau, 1643, on n'en a pas encore retrouvé un seul exemplaire.

(15) ASBR-RLPG, I Ire série, vol. 40, f. 32. PAGLIARO, p. 188.

(16) Ibid., vol. 42, ff. 92-94. PAgliaro, pp. 208-09.

(17) Sa dernière lettre de Paris est daté du 22 février 1658: cfr. La correspondance d'Albert Bailly, éd. cit., vol. VI, par L. GHIOsso, p. 254. 
la maison Saint-Eloy, à partir de $1647^{18}$, charge renouvelée en $1650^{19}$, ensuite comme visiteur général, à partir de 1653 , charge renouvelée en $1656^{20}$.

Au cours de ce long séjour parisien, le père Bailly envoya à la cour de Turin et surtout à sa protectrice Marie-Christine de France et à son premier secrétaire Guillaume-François Carron de Saint-Thomas un nombre considérable de lettres que l'on vient de publier.

Vers la fin de 1656, un événement fâcheux se produisit. On lui fit croire qu'il avait été élu évêque d'Ivrée ${ }^{21}$. La nouvelle était fausse et le père Bailly fut bien marri d'être devenu un «évêque imaginaire ${ }^{22}$, mais la première Madame Royale ne le fit pas languir trop longtemps puisque, vers la fin de l'année suivante, elle le proposa pour le siège d'Aoste ${ }^{23}$. Notre prélat quitta donc Paris pour se rendre à Rome et passer son examen $^{24}$.

Le 14 juin 1658 le duc Charles-Emmanuel II, alias Marie-Christine de France, lui faisait cadeau de cinq mille lires d'argent pour le défrayer de son voyage vers la capitale de la chrétienté et d'un anneau valant trois cents doubles d'Espagne (document III). Le 22 décembre de la même année, le trésorier général Giovanni Pietro Forneri confirmait le don de cinq mille lires d'argent «in aiuto di costa» pour Mgr Bailly, à prélever sur le donatif que le duché d'Aoste aurait fait au duc pour les années 1659. 1661(document IV). L'administration savoyarde avait l'habitude de manger son blé en herbe.

Rentré à Turin, Mgr Albert Bailly fut consacré le 9 mars 1659 dans l'église cathédrale par l'archevêque Giulio Cesare Bargera assisté par les évêques d'Asti et d'Ivrée $e^{25}$ et il fit son entrée solennelle dans son diocèse le 19 du même mois ${ }^{26}$. A peine installé à Aoste, le nouvel évêque recevait d'autres mil cinq cents livres d'argent «per degne considerationi» (document V). Il en avait bien besoin, le palais épiscopal étant complètement délabrée ${ }^{27}$. Il fallait le rebâtir ${ }^{28}$, ce qui fut fait dès $1661^{29}$.

(18) ASBR-S (Acta capitulorum generalium), vol. 28, f. 32v; R (Acta Praepositi Generalis), vol. 7, f. 26v.

(19) Ibid., S, vol. 29, ff. 15v.-20 et R, vol. 7, f. 117r.-v. S. Pagano, Gerarchia barnabitica. Prepositi generali - Assistenti generali - Procuratori generali - Prepositi provinciali - Prepositi e superiori locali, éd. cit., vol. I, p. 165, et additions à la main.

(20) Ibid., S, vol. 30, f. 23v. (première élection le 15 mai 1653 ) et vol. 31, f. $15 \mathrm{v}$. (réélection le 17 mai 1656). Pagliaro, p. 101 et n. 95.

(21) La correspondance d'Albert Bailly, éd. cit., vol. VI par L. GHIOsso, p. 117 et pp. 24-25.

(22) Ibid., p. 125

(23) Ibid., p. 252.

(24) Pour les lettres envoyées de Rome, cfr. l'édition par Luca Giachino citée dans la note 1.

(25) A.P. FruTAz, Le fonti per la storia della Valle d'Aosta, Roma, Edizioni di Storia e Letteratura, 1966 («Thesaurus Ecclesiarum Italiae», I, 1), p. 318.

(26) Ibid., pp. 318-19. J.-M. AlBini, Mémoire bistorique sur Philibert-Albert Bally, évêque d'Aoste et comte de Cogne au XVII siècle, Turin, Imprimerie de Séb. Franco et Fils, 1865, p. 32.

(27) J.-M. AlbinI, Mémoire historique sur Philibert-Albert Bally, évêque d'Aoste et comte de Cogne au XVIIe siècle, éd. cit., pp. 46-48.

(28) La correspondance de Mgr Albert Bailly, vol. VII (2005), par G. Puttero, p.79, lettre
d'Aoste du 28 mars 1659 à Marie-Christine: «Il [Jean-André Perron-Balme de Donnas] offre cent pistolles; tout de bon, Madame, si V.A.R. les vouloit donner pour rebatir cette pauvre maison de l'Evesché qui tombe en ruine: je les luy ferois multiplier au donatif de son mariage». Lettre du 26 juillet 1661 à la même (cfr. éd. Puttero citée, pp. 225-26: «V.A.R., Madame, m’ayant nommé à l'Evesché d'Aoste, pour me donner le moien d'y subsister selon ma dignité et de pouvoir en quelque façon reparer les ruines des biens de l'Evesché et du palais episcopal, que la nonchalance de mes predecesseurs, et les spoliateurs de la Chambre Apostolique avoient reduit en un pitoiable estat, elle voulut bien me gratiffier d'une pension de trois cent ducatons sur l'Archevesché de Tarentaise, à laquelle mesme, par un surcroi de largesse, elle en ajouta une seconde, un mois aprés de deux cent ducatons ...».

(29) Ibid., vol. VII. p. 370-71, lettre d'Aoste du 28 août 1661: «Je vous rends, Madame, de tres humbles graces de vostre protection toute puissante, et si cette ville peut estre quelque jour honorée de la presence de V.A.R., j’espere de luy faire voir ses bienfaits emploiez à la reparation, j'ose presque dire, à la construction d'une maison qu'elle trouvera aussi belle, et aussi commode, qu'elle luy parut, quand elle fut ici [en juillet 1656], horrible et inhabitable». Suit la description des travaux exécutés. 
Le document suivant remonte à 1667. Bien du temps avait passé. Marie-Christine était décédée le 27 décembre 1663, bientôt suivie par Françoise d'Orléans (14 janvier 1664), première femme de Charles-Emmanuel II. Ce dernier pouvait alors se marier finalement avec sa cousine Marie-Jeanne-Baptiste de Nemours (10 mai 1665), que Mgr Baillly avait connue jeune orpheline à Paris et qu'il avait toujours présenté favorablement à Marie-Christine. Après une fugace allusion à la beauté de cette jeune fille, dans une lettre du 17 avril $1654^{30}$, il brossait un portrait flatteur de cette princesse à Madame Royale le 9 juillet $1655^{31}$ et encore en $1658^{32}$, mais celle-ci faisait la sourde oreille ${ }^{33}$.

Marie-Jeanne-Baptiste n'oublia pas les efforts faits par ce Barnabite pour la placer sur le trône de Savoie et, dès qu'elle devint, à son tour, régente le combla de bienfaits.

Elle lui fit cadeau de deux mille lires d'argent en 1676 (document VI) et encore en 1678 (document VII), dont mille payées la même année (document VIII). Ces deux dernières sommes d'argent reçues par Mgr Bailly (documents IX et X) ne sont pas des gratifications mais des remboursements. Ces deux lettres patentes portent la signature de Victor-Amédée II et elles remontent à 1680. Il s'agit des pièces comptables les plus intéressantes.

Une fois installé dans son diocèse, Mgr Bailly a été fort sensible à la pauvreté du pays $^{34}$ et il déploya tous ses efforts pour y remédier. Ses lettres, où il est question de ce problème, sont trop nombreuses pour les citer toutes. Je me borne donc a en transcrire quelques-unes, parce qu'elles sont liées aux deux patentes que l'on trouvera dans l'appendice (documents IX et X). Ces documents concernent la découverte des eaux minérales de Courmayeur.

Sur ce sujet, il existe une bibliographie aussi importante que décevante ${ }^{35}$. Une

(30) Ibid., vol. V par P. Cifarelli, p. 106.

(31) Ibid., p. 249: «Madame de Nemours me fit voir, ces jours passés, Mademoiselle sa fille aisnée. En verité, Madame, elle est tres belle, de la plus riche taile du monde, et fort crüe. Il n'est point de blancheur qui aproche la siene, ella a beaucoup d'enbonpoint, et de magesté. J'ay sçeu au vray son âge, car V.A.R. me commanda expressement l'année passée, que je fisse diligence pour l'aprendre, et que je luy ecrivisse. Elle naquit donc l'onzieme avril, jour de saint Leon, de l'année 1644. Elle est nubile, et quelque difficile que soit Monseigneur, à s'engager, en verité il ne pourroit s'empescher de l'aimer, s'il la voioit. Au reste, c'est une douceur, et une bonté infinie».

(32)L. Giachino, Lettres inédites de Mgr Albert Bailly (Rome 1658), éd. cit., pp. 166-67. Dans une lettre du 30 septembre, après avoir dit bien du mal de la «Grande Mademoiselle», fille de premier lit de Gaston d'Orléans, il ajoute: «L'autre (M.-J.-B. de Nemours) a 14. ans; elle est fort jolie, et il n'est point de teint qui egale le sien; la taille en est admirable, et sa blancheur a peu de semblables. C'est un mouton, elevée dans la creinte et la vertu. Vous en ferez ce que vous voudrez; elle n'a pas trop de naissance pour s'en orgueillir, elle en a assés pour la reputation de S.A.R., car elle est princesse du sang de Savoie; et puisque V.A.R. m'appelle en riant son Prelat, vostre Prelat, Madame, serieusement vous la conseille».

(33) Ibid., p. 123, lettre du 19 août 1658: «La bonne dame (Madame de Nemours) voudroit ce que V.A.R. sçait bien qu'elle desire, et que j'y travaillasse; et moi, ne pouvant vouloir, ni solliciter que ce que V.A.R. veut que je veuille, et que je sollicite, quand ce seroit pour gagner un empire, je ne luy écris point et ne vous parle point de sa fille parceque je ne sçay que luy écrire, et que je ne debite jamais que ce que je sçay, et que V.A.R. veut bien que je sache, et que je debite».

(34) On trouvera quelques renvois dans: G. Mombello, Monseigneur Albert Bailly à travers sa correspondance inédite, «Bulletin de l'Académie Saint-Anselme», nouvelle série, II, 1987, p. 22 , n. 37 et surtout dans le vol. VII (2005) de sa Correspondance que vient de publier Mme Giorgia Puttero.

(35) Je remercie vivement M. le Dr. Omar Borettaz da la Bibliothèque Régionale d'Aoste qui a bien voulu me fournir une cinquantaine de renvois bibliographiques sur ce sujet. Je me borne à citer quelques ouvrages. Traité des eaux minérales de Courmayeur. Par Mr. Mollo [Jean-DominiqueMarie], Docteur en Médecine en l'Université de Turin, et Médecin Juré des Etats du Duché d'Aoste, Genève, Chez Marc-Michel Bousquet et Comp. MDCCXXVIII, 1-30 et passim. Analyse des eaux minérales de S. Vincent et de Courmayeur dans le Duché d'Aoste, avec une appendice sur les eaux de La Saxe, de Pré S. Didier et de Fontane-More, Par M. GionnnetTI, docteur collégié, Doyen, et Vice-Prieur de la Faculté de Médecine de Turin, médecin pensionnaire de S.M.... A Turin, chez Jean-Michel Briolo, M. DCC.LXXXX. B. BERTINI, 
recherche substantielle reste à faire, dans ce domaine assez important pour l'histoire de l'économie valdôtaine. Je propose ici une toute petite contribution.

Les historiens qui se sont intéressés à $\mathrm{Mgr}$ Bailly ${ }^{36}$, ou à cet espect de l'économie valdôtaine, ont mis en relief l'action de cet évêque pour la valorisation des sources d'eau minérale de Courmayeur. Découvertes vers le milieu du XVII e siècle, elles étaient au nombre de trois: la Victoire, la Marguerite et la Marie-Jeanne-Baptiste, plus la Saxe sise en amont du chef-lieu, dans le lieu-dit qui porte ce nom.

Dans l'avant-dernier document transcrit ici (numéro XVIII), notre évêque se flatte d'avoir «découvert ces tresors» et surtout la troisième source de Courmayeur, qu'il appelle ailleurs «nostre fonteine» (document XI).

Dans une lettre du 10 septembre 1680 (document XIV), il raconte que l'ingénieur Rubat avait assisté, en qualité de parrain, au baptême des trois sources: Victoire, Marguerite et Marie-Jeanne-Baptiste auxquelles on aurait imposé les noms de Jeanne, Baptiste et Marie, noms que l'évêque allait «confirmer» le lendemain - et lui seul pouvait le faire - en: Marie-Régente, Marie-Régnante et Marie-Bien-Faisante (document XIV). Il plaisante, bien sûr, tout en flattant sa protectrice.

Deux au moins de ces sources étaient connues dès avant 1676, lorsque le capitaine Montendon fut envoyé à Courmayeur ${ }^{37}$ pour s'enquérir de leur existence et de leur nature.

Malgré le scepticisme des «incrédules» (document XI), des «ennemis» (document XIV) et de «certaines gens - un ou deux - qui se font de leur chagrin leur plaisir et leur tiltre» et qui «la décrient cette innnocente source» (document XIII) - il s'agit de la dernière qui portait le nom de la duchesse -, Mgr Bailly se pose en son défenseur acharné.

En effet, cette fontaine avait un défaut, elle était placée trop près de la Doire. L'été 1680 avait été particulièrement chaud ${ }^{38}$ et il fut suivi par des inondations ${ }^{39}$ qui emportèrent les digues que l'entrepreneur Canova était en train de construire pour protéger la fontaine Marie. Elles furent vite réparées (document XVI). L'évêque se porta garant de l'entrepreneur auprès de la duchesse ${ }^{40}$.

Idrologia minerale, ossia descrizione di tutte le sorgenti d'acque minerali note sinora negli Stati di S. M. il re di Sardegna ... Seconda edizione accresciuta, corretta e migliorata, Torino, Dalla tipografia di Enrico Mussano, 1843, pp. 147-56. Davet De Beaurepaire, Histoire et description des sources minérales du Royaume de Sardaigne et des contrées voisines, Paris, s.d. [1852?], pp. 476. Compte rendu par B. Bertini in «Giornale della Reale Accademia Medico-Chirurgica», VIe année, vol. XVII, n 17,10 juin 1853 , pp. 238-44. G.-A. GIUSTA, Guida ai bagni ed alle acque minerali, solforose, alcaline, iodurate e bromurate ed a quelle ferromagnesiache ed acidole di Courmayeur, con alcuni cenni sulle terme di Pré-Saint-Didier, Aosta, Luigi Mensio, 1875, pp. 22-23. V. SANTANERA, Brevi cenni sulle acque minerali e in particolare sulle acque di Courmayeur e Pré-Saint-Didier nella Valle d'Aosta. Nuova guida pratica, Torino, F. Casanova, 1879, T. TIBALDI, Brevi cenni storici sulla sorgente JeanneBaptiste in Cournayeur e sul nuovo stabilimento idroterapico di Michel Tavernier, Aosta Tipografia Luigi Mensio, 1884, plaquette de quinze pages. S. BALP, Le sorgenti minerali della provincia di Torino, Perugia, Unione Tipografica Cooperativa, 1902, pp. 33-40. Mgr J.-M. Duc, Histoire de l'Eglise
d'Aoste, Châtel-Saint-Denis, Imprimerie Moderne, 1901-1916, en 10 voll., t. VII, pp. 362-63. G. IssoGLIO, Le acque minerali di Courmayeur, in «Annali della R. Accademia di Agricoltura di Torino», vol. sessantasettesimo, 1924 (Torino, Tipografia Enrico Schioppo, 1925), pp 3-17. J.-M. HENRI, Histoire populaire, religieuse et civile de la Vallée d'Aoste, la première et la plus antique terre du Royaume d'Italie, Aoste, 1929, pp. 291-92. Je n'ai pu par contre consulter: Sorgenti e terme in Valle d'Aosta: un percorso fra storia e religiosità, immaginario e scienza. A cura di A. Peretti, Aosta, Musumeci, 2000.

(36) Par exemple, Mgr. J.-M. Duc et l'abbé J.-M. Henri sans leurs ouvrages cités dans la note précédente.

(37) Mgr J.-M. Duc, Histoire de l'Eglise d'Aoste, op. cit., p. 362 et n. 1. J.-M. HENRI, Histoire populaire, religieuse et civile de la Vallée d'Aoste, la première et la plus antique terre du Royaume d'Italie, éd. cit., p. 291.

(38) Deuxième texte du document XIV: «Nous avons ici l'été, mais si chaud qu'on y brûle».

(39) Mgr J.-M. Duc, Histoire de l'Eglise d'Aoste, éd. cit., p. 365 .

(40) Cfr. les deux pièces transcrites sous le numéro XIV. 
Mgr Bailly lança aussi une véritable campagne de promotion, tout d'abord en donnat la listes des curistes qui avaient déjà profité des eaux de Courmayeur, parmi lesquels il cite le doyen de la Saint-Chapelle de Chambéry François Bertrand de La Pérouse, le gardien des capucins de Saluces (document XII), le marquis, la marquise et l'abbé de Canei(?) (documents XIII, XV et XVII), la comtesse d'Osasco, ou d'Osasio (document XIV) et des hydropiques (document XIX). Ensuite il se fait fort des avis émis par des spécialistes reconnus: le capitaine Montendon (deuxième pièce du document XIV), le médecin Gaspare Antonio Ravetti et le chimiste Campeggio (document XI et la deuxième pièce du document XIV) et le protomédecin Torrini (document XVII) pour appuyer sa conviction de la bonté de ces eaux. Dans les deux pièces du document XIV, il communiquait à la duchesse que les digues seraient vite réparées et que des logements confortables seraient «prêts et suffisants» soit à Courmayeur soit à Morgex, où on aurait pu trouver, dans la maison du secrétaire du duché Eugène-Gaspard de Tillier, vingt-cinq belles chambres.

Que le protomédecin Torrini lance donc son «manifeste» (document XVII) ${ }^{41}$ ! Quant à lui, il fait des vers pour un «cartel hardi» qui chante les vertus de la fontaine Marie (pièce XIII). Il n'attend plus que de pouvoir placer, auprès de cette fontaine la statue de la duchesse munie d'une inscription, le tout aux frais de la princesse, bien entendu; bref il se pose en véritable agent de publicité.

Malgré tous ses efforts, la fontaine qui portait le nom de la duchesse n'existait plus déjà au début du XVIII e siècle, emportée par la Doire dont elle n'était que trop proche $^{42}$. Vers la fin du XIX ${ }^{\mathrm{e}}$ siècle, un Valdôtain qui avait fait fortune à Paris: Michel Tavernier, pensa l'avoir retrouvée et on l'appella source «Regina» ${ }^{43}$. En 1883, il ouvrit un établissement hydrothérapique qui eut du succès ${ }^{44}$, mais aujourd'hui il n'existe plus aucun établissement de ce genre dans cette localité très courue en hiver comme en été.

C'est une raison de plus pour rappeler, à la mémoire des Valdôtains et de ceux qui ont une prédilection pour cette ville de vacances, un épisode qui a eu et qui garde un certain intérêt dans l'histoire économique de l'ancien duché d'Aoste. Encore une fois, Mgr Albert Bailly a joué un rôle non négligeable dans cette affaire. En sus de ses autres mérites, il faudra donc lui reconnaître aussi celui d'avoir été, au XVII siècle, un pionnier convaincu de l'hydrothérapie dans la Vallée d'Aoste.

GIANNI MOMBELLO

\section{DOCUMENTS}

I

AST, Sezioni riunite, Patenti controllo finanze, registre $1638,2^{\circ}$, f. $97 \mathrm{r}$.

Il duca di Savoia.

Camera nostra de conti di qua da' monti. Entrate e fatte buono in quelli del tesoriere nostro di militia messer Giovanni Ferrero, la somma di ducatoni ducento da

(41) J.-D.-M. Mollo parle de ce «manifeste» du docteur Torrini, que je n'ai pu retrouver, aux pp. 28-30 de son Traité des eaux minerales de Courmayeur, éd. cit.

(42) Cfr. la p. 10 de l'ouvrage de Jean-Dominique-Marie Mollo cité dans la note 35.

(43) Il n'est pas prouvé que la source «Regina» corresponde exactement à celle qui avait été découverte du temps de Marie-Jeanne-Baptiste. Cfr. $\mathrm{G}$. Issoglio, Le acque minerali di Courmayeur, op. cit., p. 15.

(44) Cfr. la plaquette de Tancredi Tibaldi citée dans la note 35 . 
fiorini tredici l'uno che ci ha sborsati in proprie mani e da noi fatti distribuire, cioè: ducatoni .85 . simil[mente] in una vesta da camera d'armellino per nostro servitio, ducatoni 15 . fatti dare per l'elemosina, ducatoni 25 . fattone accomprar caratiglie ${ }^{45}$ per il baletto di Madama Serenissima, ducatoni 50. fatti dare al secretaro Bailly, e ducatoni 25. fattone accomprare tanti fiori di Riviera, de qual ducatoni 200. come sopra vogliamo che con questo senza altro resti detto tesoriere appresso di voi scaricato in detti suoi conti, sí come noi sin d'hora ne lo discarichiamo, ché cosí ci piace. Dato in Torino, li 29. genaro 1629. Carlo Emanuele, $v^{a}$. Piscina, $v^{a}$. Buonfiglio presidente, $v^{a}$. Pellegrino.

Dans la marge. Secretaro Bayli

Ibid., registre $1633, I^{\circ}$, f. 68 r.

Il duca di Savoia, re di Cipri.

$\mathrm{Al}$ magnifco consigliere et tesoriere nostro generale messer Francesco Verdina presente e succesori, salute. Vi ordiniamo con le presenti che di qualsivogli danaro più liquido di vostra riccetta niuno eccettuato dobbiate assignare, pagare, o far pagare al Reverendo Don Alberto Baly la somma di ducatoni trecento effettivi da soldi quarantasei d'argento l'uno, cio è livre cento d'argento, a soldi 20. l'una in contanti prontamente, et il restante sino al compimento di detti ducatoni 300. in tante stoffe sopra il primo partito che si farà di esse con noi, al quale gli facciamo dare per impiegarli in un oppera pia, che rittenendo nel primo pagamento copia autentica delle presenti con quittanza di detto Reverendo Bally, o d'un suo legitimo agente, e nel finale le presenti con quittanza come sopra, vogliamo ne resti detto tesoriere scaricato ne suoi conti, per detta somma dalla Camera nostra d'essi, alla quale madiamo di cosí fare, et osservar senza difficultà alcuna, qualonque ordine, bilancio, o altro in contrario che ripugnasse, ché tal è nostra mente. Datto in Torino a 20 d'agosto 1633. Vittorio Amedeo. V'. Piscina - Va. Furno presidente - V'. Gentile - Carraro

Dans la marge.

D. Alberto Baly, ducatoni 300. per impiegarli in una opera pia.

Ibid., registre 1658, f. 116v.

Il duca di Savoia, etc.

Camera nostra de' conti di qua da' monti. Passate et entrate in quelli del magnifico consigliere e tesoriere nostro generale messer Giovanni Pietro Forneri la somma di livre cinque milla d'argento, a soldi 20. l'una per altretante ch'egli d'ordine nostro verbale ha pagate al molto reverendo padre Don Alberto Bally eletto vescovo d'Auosta, al quale ne abbiam fatto dono in aiuto di costa, per supplire alle spese che deve fare nel viaggio di Roma per l'admissione al sudetto vescovato. Delle quali lire 5000. d'argento come sopra pagate, vogliamo che con questo et quittanza di detto

(45) Par cartiglia on pouvait indiquer, soit une feuille de papier avec notation musicale, soit

une carte à jouer. De l'espagnol «cartilla». 
molto reverendo padre Bally senz'altro ne venga il sudetto tesoriere generale Forneri sufficientemente discaricato ne' suoi conti appresso noi, come noi sin d'hora ne lo discarichiamo. Ché tal'è nostra mente. Dato in Torino, li 14. di giugno 1658. Firmato: Carlo Emanuele, va. Morozzo, va. Turinetti e, per il Generale, sottoscritto Sansoz.

Dans la marge.

Per il padre Bally eletto vescovo di Auosta, discarico di lire 5000. argento, per aiuto di costa.

Si pagano qui dietro per un'anello (sic) con diamante dato al sudetto padre Bally, doppie 300. Spagna.

Ibid., registro 1658 in 1659 , f. $104 \mathrm{r}$.

IV

A dí primo febraro 1659 .

Giovanni Pietro Forneri tesoriere generale.

Confesso haver havuto et ricevuto dal ducato d'Auosta, et per le mani del signor Giovanni Bonifaccio Festa suo tesoriere, la somma di livre cinque milla d'argento, a soldi 20. l'una quali sono a conto del donativo che si fa a S. A. R. nelli tre anni prossimi 1659. 1660. e 1661, pagarà, ne due primi quarti o semestri, al molto reverendo padre Don Alberto Bally eletto vescovo di Auosta per donativo fattogli in aiuto di costa, et per supplir alle spese del suo viaggio di Roma per l'admissione del sudetto vescovato, et ciò in virtù di discarico di S.A.R. delli 4 (sic) giugno prossimo passato. De' quali lire $5 / \mathrm{m}$. comesse quitto detto ducato, signor tesoriere Festa, et chi spetti. Torino, li 22. Xbre 1658.

Sottoscritto Giovanni Pietro Forneri

Per lire 5000.

Dans la marge:

Per debito al tesoriere generale Forneri di livre 5000. d'argento.

Ibid., registre 1658 in 1659 , f, 175 r.

Il duca di Savoia, etc.

Camera nostra de conti di qua da monti. Passate in quelli del magnifico consigliere e tesoriere generale nostro Giovanni Pietro Fiorneri la somma di livre mille cinquecento d'argento, a soldi 20. l'una per altretante ch'egli del denaro del donativo, che di presente ci fa il ducato d'Auosta ha, d'ordine nostro, pagato al vescovo del detto ducato D. Alberto Bally al quale noi ne facciamo dono per degne considerationi del servitio nostro. Delle quali livre 1500. d'argento come sopra vogliamo che il detto tesoriere generale mediamte il presente e la contenta d'esso vescovo resti presso di noi scaricato in detti suoi conti, come noi sin d'hora ne lo scarichiamo, perché cosí ci piace. Datto in Torino, li 23. maggio 1659.

Firmato: Carlo Emanuele, va . Binelli pro domino Cancellario, vª Turinetti, v ${ }^{a}$. Trabucco, sottoscritto de Saint Thomas.

Dans la marge:

Per Monsignor vescovo Bally, discarico di livre 1500. d'argento in donativo. 
Ibid., registre $1676, \mathrm{I}^{\circ}$, f. $33 \mathrm{v}$.

La duchessa di Savoia, etc.

Camera de conti di S.A.R. di qua da monti, passate in quelli del magnifico consigliere tesoriere generale Messer Giovanni Matteo Belli la somma di livre due milla d'argento, a soldi 20. l'una per altretante ch'egli ci ha sborsate in proprie mani per servitio secreto di S.A.R., de quali livre $2 / \mathrm{m}$. come sovra vogliamo che con questo senz'altro ne resti detto tesoriere generale Belli sufficientemente scaricato ne suoi conti appresso di noi, omessa qualonque difficoltà, come noi sin d'hora ne lo scarichiamo, che tal'è nostra mente. Dato in Torino, li 3. aprile 1676.

M. Jeanne Baptiste, $v^{a}$. Buschetto, $v^{a}$. Truchi, $v^{a}$. Ferrari.

Postilla mia. Mediante quittanza di Mosignor vescovo d'Auosta.

Sono livre 2000. sborsate come sovra in mani di V.A.R. e da lei donate al vescovo d'Auosta Baly per degne cause l'animo suo moventi ${ }^{46}$.

Dans la marge:

Vescovo d'Auosta Baly, livre 2000.

Ibid., registre 1678, f. $163 \mathrm{v}$.

La duchessa di Savoia, etc.

Camera de conti di S.A.R. di qua da Monti. Passate et entrate in quelli del magnifico consigliere e mastro auditore in cotesto magistrato Silvestro Olivero ${ }^{47}$, amministratore deputato alla tesoreria generale per il tesoriere generale Spirito Francesco suo figliolo, la somma di livre mille d'argento, a soldi 20. l'una per altre tante ch'egli del danaro del donativo presentemente aggiustato et dovuto dal ducato d'Auosta, in sei anni venturi, da principiare subito spirati li termini de pagamenti del donativo antecedente et in dodeci semestri repartitamente pagarà, al vescovo di detta città d'Auosta, al quale n'habbiamo fatto dono per le considerationi a noi riservate. De quali livre 2000, come sopra, vogliamo che con questo, la quittanza di detto vescovo, senz'altro ne resti ditto auditore et amministratore appresso di noi sufficientemente scaricato ne suoi conti, ommessa qualunque difficoltà, come noi sin d'hora ne lo scarichiamo. Che tal'è nostra mente. Dato in Torino, li 23. agosto 1678.

M. J. Baptiste, va. Simeone pro domino Cancellario, va. Truchi, va. Garagno, sottoscritto Giordano.

Dans la marge:

Lire 2000. Vescovo d'Auosta, dono.

(46) Mgr Bailly s'empressa de remercier la duchesse par une lettre datée de «juin 1676» dont voici le début: «Madame, je rends de tres humbles graces à V.A.R. du present qu'elle a daigné me faire de 2000 $£ £$ que Mr. le General de finances m'a fait paier ici comptant. Il est plus obligeant et plus adroit que celui qui, pour avoir porté en longueur le donatif de 200 ducatons que feu M.R. m'avoit acordé, m'obligea, comme je me suis doné l'honeur de vous écrire, de lui remettre le papier et fit que cette genereuse maitresse m'en fit paier comptant le tiers de plus et me l'envoia avec une bague de diamants par Mr. le general Ferraris intendant de votre maison et mon ancien ami. J'ay dit, Madame, que Mr. le General de finances a eu plus de conduite que l'autre, car il a bien preveu que, s'il eut tardé à me faire paier, V.A.R. auroit doublé la charité et Messieurs les financiers sont et doivent estre contre les largesses des princes, qui ne sçauroient rien refuser. Serieusement, Madame, je suis tres satisfait et je ne lui demande plus rien pour moi. Il est bien vrai que, quand je continuerois de demander, je ne ferois que ce que V.A.R. m'a depuis tres longtemps ordoné de faire, mesme sans hesiter, n'en deplaise aux critiques...». AST. Lettere vescovi. Aosta, liasse 2, fasc. 1, pièce 47.

(47) Ce nom a été répété deux fois par mégarde. 
Ibid., registre 1678 in 1679, f. 50.

\section{VIII}

Silvestro Olivero consigliere e mastro audiore, etc.

Confesso haver havuto et ricevuto dal Signor Grato Migliore tesoriere del ducato d'Auosta per S.A.R. la somma di livre mille d'argento, a soldi 20. l'una, in tante che a conto del donativo offerto a detta R. A. dal medemo ducato, pagabili in sei anni et reppartitamente in dodeci semestri, il primo de quali terminerà li 15 genaro dell'anno 1680, pagarà a Monsignor illustrissimo vescovo di detta città d'Auosta per dono fattogliene M. R \per cause/ alla medema R.A. riservate, in virtù di suo discarico delli 23. agosto prossimo scorso. De quali livre 2000. come sopra quitto detto signor tesoriere Migliore et chi spetti. Torino, li 20. 9bre 1678.

Silvestro Olivero.

Per livre 2000.

Dans la marge:

Tesoriere Migliore

Vescovo d'Auosta

In debito all'auditore Olivero.

Ibid., registro $1680, \mathrm{I}^{\circ}$, f. 151.

\section{IX}

Il duca di Savoia, etc.

Camera nostra de conti. Passate in quelli del magnifico consigliere e mastro auditore Silvestro Olivero, amministratore della tesoreria generale per il tesoriere generale suo figliuolo, la somma di livre mille cinquecento d'argento per altre tante ch'egli ci ha sborsate e noi habbiamo fatte dare al vescovo d'Agosta, cioè, livre 1300. in rimborso d'altra simil somma dal medemo spesa attorno la fabrica che si fa alla fontana vitriolata ${ }^{48}$ e livre 200. per altre spese straordinarie e massime per la speditione di pedoni, dalla prova di che tutto lo liberiamo per esserne noi appieno informati; de quali livre 1500. d'argento, come sopra, vogliamo ch'il detto auditore et amministratore Olivero, mediante il presente e la quittanza d'esso vescovo d'Agosta, senz'altro resti appresso di noi sufficientemente discaricato ne' suoi conti, come noi sin d'hora ne lo discarichiamo. Che tal'è nostra mente. Dato in Torino, li 5 . giugno 1680.

Vittorio Amedeo, va. Simeone pro domino Cancellario, va. Truchi, va. Garagno. Aymone, etc.

Dans la marge:

Vescovo d'Auosta.

Ibid., registre 1680 , $2^{\text {e }}$, f. 220r.-v.

\section{$\mathrm{X}$}

Il duca di Savoia, etc.

Camera nostra de conti. Passate in quelli del magnifico consigliere e mastro

(48) Cfr. le texte que Mgr Bailly a ajouté à sa lettre du 10 septembre 1680 et transcrit ici sous le numéro XIV. Dans ce cas, il ne s'agit pas d'un cadeau, mais d'un remboursement. 
auditore Silvestro Olivero, amministratore della tesoreria generale per il suo figliuolo, la somma di livre mille novanta d'argento che egli ci ha sborsate e noi habbiamo fatto dare al vescovo di Agosta in rimborso di simil somma dal medemo spesa attorno la fabrica della fontana, o siano bagni ${ }^{49}$ della valle di Agosta, oltre le livre 1500. già pagate a detto vescovo, dalla cui prova lo liberiamo per esserne noi pienamente informato. De quali, livre 1090, come sovra, vogliamo che il detto auditore et amministratore Olivero, mediante il presente e la quittanza d'esso Vescovo d'Agosta, senz'altro resti appresso di noi sufficientemente discaricato ne suoi conti, come noi sin d'hora ne lo discarichiamo. Che tal'è nostra mente. Dato in Torino, li 23. $7^{\text {mbre }} 1680$.

Vittorio Amedeo, va. Simeone pro domino Cancellario, va. Truchi, va. Garagno, etc.

\section{Dans la marge.}

Vescovo d'Agosta.

\section{XI}

AST, Sezione corte, Lettere vescovi. Aosta, liasse 1, fasc. 7 avec cette inscription récente. «Lettere di vescovi - Aosta, 1659-1675. Lettere scritte al Duca ed la marchese di S. Tommaso da Alberto Baylly di Geneva Vescovo di Aosta». Pièce 243, datée «17 septembre», sans millésime [1687?]. Le destinataire est Marie-Jeanne-Baptiste de Nemours.

\section{Madame,}

les deux habiles visiteurs ${ }^{50}$ de nos eaux s'en vont bien satisfaicts de leur voyage. V.A.R. pourra se faire une petite et agreable recreation de tout ce qu'ils vous diront de la vertu et de la beauté de nostre fonteine, qui attend impatiemment votre statue pour en faire son ornement et sa decoration. Une des Naiades qui s'y baignent m'est venu dire de la part des autres, que sans les prieres que nous faisons pour votre santé, elles auroient esperé que Dieu auroit exaucé celles qu'elles lui font de vous faire souffrir une legere infirmité et dont vous ne puissiés guerir que par l'usage des ces eaux, pour avoir l'honeur de vous voir et de vous adorer. Au moins, Madame, j'ai la consolation de faire avouer à quelques incredules, que l'eveque d'Aoste, par son caractere et par sa propre et naturelle inclination, ne peut pas faillir de dire la verité, sur tout quand il s'agit de l'obligation qu'il a de ne rien proposer à une souveraine, qui ne soit presque TEXTE D'EVANGILE. Je suis tres respectueusement, Madame, de V.A.R. tres humble, et tres obeissant sujet, et serviteur. D. Albert, E. D'Aoste.

Ce 17. de sept[embre].

(49) Cette précision mérite d'être retenue. L'eau de la fontaine Marie-Jeanne-Baptiste devait donc être au moins tiède. Malheureusement je n'ai pu retrouver les descriptions qu'en ont données $G$. A. Ravetti, Campeggio et Bartolomeo Torrini, mais Mollo (Traité des eaux minérales de Courmayeur, éd. cit., p. 29), en citant Torrini, parle de la «chaleur» de la Marguerite et de la Victoire. La source redécouverte par Michel Tavernier, à la fin du XIXe siècle, n'avait que 8,7 degrés. Cfr. G. IssogLIo, Le acque minerali di Courmayeur, éd. cit., p. 13.

(50) Il s'agit du médecin Gaspare Antonio Ravetti et du chimiste Campeggio qui avaient été envoyés à Courmayeur en 1687. Ils ont publié leur Analyse des eaux de Courmayeur à Turin la même année. Cette lettre doit donc remonter à cette année-là. Cfr. la pièce publiée ici sous le numéro XVII. Pour G.A. Ravetti, cfr. G.G. Bonino, Biografia medica piemontese, Torino, Dalla tipografia Bianco, 1824-1825, pp. 439-40. GIUSTA, Guida ai bagni ed alle acque minerali, solforose, alcaline, iodurate e bromurate ed a quelle ferro-magnesiache ed acidole di Courmayeur, con alcuni cenni sulle terme di Pré-Saint-Didier, éd. cit., p. 22, n. 1 et T. TIBALDI, Brevi cenni storici sulla sorgente Jeanne-Baptiste in Courmayeur e sul nuovo stabilimento idroterapico di Michel Tavernier, op. cit., p. 7, n. 1. Malheureusement je n'ai pu retrouver aucun exemplaire de l'Analyse de Ravetti et Campeggio. 
XII $1679 ?)^{51}$

Ibid., pièce 285 à la même, datée «8 aoust», mais sans millésime (avant octobre

\section{Madame,}

j'ai eu l'honeur de loger Mr. l'abbé de La Perrouse ${ }^{52}$ à son retour des eaux de Cormaieul. J'appelle cet honeur precieux, particulierement parce qu'il m'a donné la satisfaction d'aprendre de la bouche de ce digne Abbé tant devoué à votre service, que V.A.R. daignoit bien continuer d'avoir pour moi ses ancienes bontés, dont je vous rends, Madame, de tres humbles graces de toute l'etendue de mon coeur, que vous apellés «le bon coeur».

Il pourra vous dire cet Abbé l'excellente vertu de nos eaux minerales, aussi bien que le Pere Gardien du couvent des Capucins de Salusses ${ }^{53}$, grand medecin, qui les appelle «richi tesori». Elles vous doivent, Madame, ces eloges, car vous les avez fait conoitre par vos liberalités. Elles vous reconoitroient sans doubte, si vous leur faisiés la grace de venir recevoir leurs remerciments, ou que vous les envoiassiés querir pour vous les faire et vous n'auriez pas plustost gouté les douceurs de leurs compliments, que votre santé s'en trouveroit extremement fortifiée.

Je prie Dieu à tout moment qu'elle soit invulnerable, cette precieuse santé, come je suis tres respectueusement, Madame, de V.A.R. tres humble et tres obeissant servieur et sujet.

Le pere Albert.

Ce 8 Aoust.

\section{XIII} 1680.

AST, Sezione corte, Lettere vescovi. Aosta, liasse 2, fasc. 1, lettre 138 du 17 juillet

Madame,

votre fonteine rend de tres humbles actions de graces à V.A.R. de la bonté qu'elle a eu de commander à Madame la marquise de Canei (? $)^{54}$ de venir boire ses

(51) Cfr. la note 53 suivante.

(52) L'abbé François Bertrand de La Pérouse (baptisé le 18 juillet 1635 et mort à Chambéry le 23 avril 1695) était le fils de François, premier président de la Chambre des Comptes de Savoie mort le 24 août 1683. Prieur de Saint Baldolf (23 avril 1650) et de Chindrieux (6 septembre 1650), il fut admis à Saint Sulpice en 1656. Docteur de Sorbonne en février 1665, il fut nommé doyen de la Sainte-Chapelle de Chambéry et abbé de Payerne le 20 juillet 1665 . Cfr. E.-A. DE Foras, Armorial et nobiliaire de l'ancien duché de Savoie, continué par le comte de MARECHAL, le comte de VIRY et le baron d'YVOIRE, Grenoble, Allier Père et Fils, 1863-1938, en 6 voll., t. II, p. 194. Le père Bailly envoya un rapport détaillé sur son «acte» au Collège de Navarre à Marie-Christine de France le 23 juillet 1655 . Cfr. La correspondance d'Albert Bailly, éd. cit., t. V, par P. CIfarelli, pp. 254-56. Malheureusement les lettres que cet Abbé a envoyées à la cour de Turin s'arrêtent à l'an 1669 , ce qui nous prive d'un document éventuellement utile pour dater la lettre que je transcris. AST, Lettere particolari, liasse 76 de la lettre $\mathrm{B}$, fascicule à son nom. Ses lettres vont de 1658 à 1669 .
(53) Je remercie vivement le père Ferruccio, responsable des Archives des pères Capucins de Turin (sigle: APCT), conservé dans le couvent de l'église de Santa Maria del Monte dei Cappuccini, pour son extrême obligeance. C'est grâce à lui que j'ai pu retrouver le nom de ce père gardien.

Les Acta provinciaze Pedementanæe sont classés sous la cote: APCT, 1.3,1. D'après les recherches que le père Ferruccio est train de mener sur ces documents, il ressort que le gardien du couvent Saint-François de Saluces, dont il est question dans cette lettre, était Giovanni da Saluzzo. Il prit l'habit de Saint François le 4 août 1658 et il prononça sa profession le même jour de l'année suivante. Avant son entrée chez les Capucins, il était médecin.

Il a été par trois fois gardien du couvent de Saluces, dès décembre 1677 à octobre 1679 et ensuite dès 1684 à 1688 et dès 1692 à 1695 . Ceci nous permet de dater cette lettre de Mgr Bailly approximativement avant octobre 1679.

(54) Sur l'extrait d'une lettre que le Marquis de Canei (?) avait écrite à Mgr Bailly à propos des eaux de Courmayeur, cfr. la lettre 122 transcrite ici sous le numéro XVII et l'ouvrage de Mgr J.-M. Duc, 
eaux minerales et de lui faire esperer qu'elle y trouveroit un remede specifique contre ses indispositions. Elle a bien besoin, Madame, votre fonteine du secours que vous daignés lui prêter; de certaines gens, un ou deux, qui se font de leur chagrin leur plaisir et leur tiltre, la decrient, cette innocente source autant qu'ils peuvent, mais elle ne se rebute point et, soutenant avec intrepidité toutes leurs attaques sous l'honeur de votre protection, elle a invité tous les malades à venir chercher chez elle leur santé par un cartel hardi, dont elle prend la hardiesse de presenter une copie à V.A.R. et la voici en forme de madrigal.

Venez boire mes eaux, si vous aimés la vie!

Malades l'on guerit, si tost qu'on en a bû;

C'est que la divine Marie,

En me donant son nom, m'a doné sa VERTU.

Elle croit, Madame, cette fille unique de V.A.R. que vous lui ferez, s'il vous plaist, la grace d'agreer sa genereuse liberté et moi qui suis le secretaire de cette Naiade, j'espere que vous me permettrés bien de contresigner sa lettre et de me dire, avec mes anciens et profonds respects, Madame, de V.A.R. tres humble et tres obeissant sujet et serviteur.

D. Albert E. d'Aoste

Ce 17. de juillet 1680 .

\section{XIV}

Ibid., lettre 136 du 10 septembre 1680. Deux feuillets encartés. Seuls l'ajout du f. 1v., la formule de congé, la signature et la date sont de la main de Mgr Bailly. Les deux feuillets qui suivent sont de la main du secrétaire qui a écrit le premier bifeuillet et autographges seulement à partir du f. 2r. («Le capitaine Montendon...»)

\section{Madame,}

Le $\mathrm{S}^{\mathrm{r}}$. Rubat ${ }^{55}$ s'en retourne bien content de nos trois fonteines et de nos digues qui ne sont pas en si mauvais etat que leurs enemis auroient souhaité. Il en fera une juste relation à V.A.R.

On les batisa ces trois Naiades en la presence de cet ingenieur, qui en fut le parrein. Il imposa à la premiere le nom de Jeane, celui de Baptiste à la seconde et appella par excellence la derniere Marie, parce qu'elle est la bien-faisante et qui n'exclut persone de la precieuse et salutaire effusion de ses graces que l'eau represente. Je les confirmei le lendemain ${ }^{56}$ et les nomei les trois Maries: sçavoir, la premiere, Marie Regente, pour avoir eu le bon-heur comme de naistre durant vôtre (sic) regence, la seconde Marie Regnante, car V.A.R. regne heureusement et la troisieme Marie BienFaisante, par la santé qu'elle rend à tout le monde.

Trois fonteines qui n'en font qu'une,

Simbole de la Trinité,

Où l'on voit une deité

Uniquement se rendre à trois suposts commune.

Vous imitez, divine image,

Histoire de l'Eglise d'Aoste, éd. cit., p. 362, où toutefois de patronyme est lu «Cauly».

(55) Il s'agit de l'ingénieur Rubat. L'AST ne conserve pas de lettres de ce personnage dans la série Lettere particolari, liasse 66 de la lettre R.
(56) Mgr Bailly aurait-il voulu se rendre à Courmayeur à cette occasion? J'en doute fort, parce qu'il dit, un peu plus loin, qu'il ne peut pas marcher et que les porteurs l'ont laissé en plan. Notre évêque plaisante donc sur cette «confirmation» imaginaire. 
Les traits de vôtre (sic) original,

Pour nous afranchir de tout mal,

Vous etes comme un Dieu, puissante, bone et sage.

Vous conoissez le miserable,

Vous pouvez remplir son besoin,

Vos bontés en prenent le soin,

Pourriez-vous à moi seul n'etre pas favorable?

Dieu n'est point partial. Pour l'imiter vous ne devez pas l'etre, belle et salutaire fontaine. Vous ne l'etes point aussi, me dites vous, et qu'il ne tient qu'à moi de partager les avantages que les autres malades reçoivent de vos eaux. Cela est bien, mais je ne puis marcher et les porteurs, qui m'avoient tant promis de me mener à vôtre source, m'ont manqué de parolle. Vous me repondez que je puis les faire porter chez moi et qu'elles sont aussi bones transportées, que prises sur les lieux. D'accord, j'ai suivi votre conseil et prié mes amis, du moins je les croiois tels, de remplir des bouteilles de vos precieuses liqueurs et de me les aporter. Ils m'en ont presenté et assûré qu'elles etoitent pleines de vos eaux, je les ai crû, mais soudain qu'elles ont eté debouchées, au lieu d'une eau veritable, je n'y ai trouvé qu'une eau benite de cour qui s'est soudain evaporée. Ainsi je suis tousjours malade.

Voila, Madame, le pitoiable sort du pauvre pere Albert.

Il pourra changer. Il est entre les mains de Dieu en qui je suis avec des profonds respects, Madame, de V.A.R. tres humble et tres obeissant sujet et serviteur.

D. Albert E. D'Aoste.

D'Aoste, ce $10^{\mathrm{e}}$ de $7^{\text {bre }} 1680$.

En baut du f. 1v. Mgr Bailly a ajouté de sa main.

Les theologiens atribuent au Pere Eternel la puissance, la sagesse au Fils et au S. Esprit la bonté.

\section{Deuxième feuillet encarté}

J'ai paié deux mille et trois centz livres à l'entrepreneur Canove et on ne lui en doit plus que deux centz. Le $\mathrm{S}^{\mathrm{r}}$. Rubat pourra dire à V.A.R. que le domage que le dernier deluge a causé aux digues est peu de chose et que l'entrepreneur n'en est pas reponsable, mais bien plustost celui qui ecrivit à la cour que ces eaux avoient fait du mal à quelque persone et qu'elles ne valoient rien, ce qui fit retarder l'ouvrage. Cependant Monsieur le president Palavisin ${ }^{57}$, etant ici, aprit la bonté de nos eaux et rebutant en pleine table, où j'etois, l'escrivain qui etoit encore contre cette salutaire fonteine, il convint avec l'entrepreneur et le chargea d'executer le dessein du S'. Rubat, moie-

(57) Le marquis Tommaso Adalberto Pallavicino est né à Turin le 30 août 1647 et mort dans la même ville le 8 novembre 1717 . On peut suivre sa carrière dans l'administration savoyarde grâce aux Patenti controllo finanze des Sezioni riunite de l'AST: registre 1670 in 1671, f. 37 (sénateur au Parlement de Nice durant trois ans, ensuite dans celui de Turin), registre 1673 in $1674, \mathrm{f}, 16$ (gratification), registre 1674, f. 126 (traitement), registre 1676, 2e, f. 226 (lires 150 «per servitio secreto»), registre $1677,1^{\circ}$, f, 8 (président de la Chambre des Comptes, auditeur et surintendant général des armées), registre
1677, $1^{\circ}$, f. 192 (auditeur et surintendant général de l'artillerie), registre, 1677, 2e, f. 147 (troisième président de la Chambre des Comptes), registre 1677, 2e, f. 216 (troisième président du Sénat de Piémont), registre 1677 in 1678,f. 1 (second président de la Chambre des Comtes de Piémont), registre 1687 in 1696, ff. 35v.-36r. (chargé, le 15 janvier 1691 , d'organiser les quartiers d'hiver), etc. Bien d'autres documents attestent sa carrière successive. Résumé dans A. MANNO, Il patriziato subalpino, éd. cit., t. XIX, p. 37. Cfr. aussi: Mgr J.-M. Duc, Histoire de l'Eglise d'Aoste, éd. cit., pp. 360-62. 
nant deux mille et cincqcents livres. On detourna celui qui avoit promis de cautioner l'entrepreneur pour soutenir la passion qu'on avoit de rendre ces eaux inutiles et ce malheur seroit arrivé si je n'eusse promis pour l'entrepreneur. Le mal est que ce retardement fut cause que la chaux n'aiant pas pû assez tost se lier, se seicher et affermir les murailles, l'impetuosité du torrent, qui ne fut jamais si enflé, a penetré dans le pan le plus avancé des digues et delaiant la chaux fraische, l'a abatu, outre que l'entrepreneur n'eut pas le temps d'armer cette partie de la digue de quantité d'aix qu'il avoit preparés et que le torrent a emportés ${ }^{58}$. Enfin, le Saint Esprit dit qu'on ne doit pas conari contra ictum fluminis. C'est un cas d'ovaille et fortuit qu'on n'a pas pû eviter. On y remediera sûrement. Le $\mathrm{S}^{\mathrm{r}}$. Rubat en dira les raisons. Tousjours sans ces digues commencées, la premiere inondation auroit couvert la fonteine Marie et, ostant le moien au monde d'en boire, il n'y seroit venu persone et on n'auroit plus parlé de ces eaux. Il y a encore un nouveau motif qui rend l'entrepreneur digne des largesses de V.A.R., c'est qu'il a fait la decouverte des autres fontaines. Je me remets à la relation de Monsieur le president Palavisin de plusieurs autres choses que je lui ai ecrites sur ce sujet.

La capitaine Montendon entend en perfection l'esprit et toute les qualités des eaux minerales. Le $\mathrm{S}^{\mathrm{r}}$. Ravet, medecin, me l'a confessé. V.A.R. pourroit le mander et sçavoir ses sentiments meme sur les ouvrages à faire. Il en menageroit la dêpence. Il dit que les eaux vitriolées qu'on a trouvées à la vallée de $\operatorname{Bros}^{60}$ ne sont pas sûres, parce qu'elles sont melées de cuivre. Il espere trouver, dans ma terre de Cogne, des fonteines de sel, comme il assûre qu'il y a des mines fort fines et abondantes.

Nous avons ici l'eté, mais si chaud qu'on y brule. Ce seroit le vrai temps de boire nos eaux. J'ai commencé ce mattin d'en prendre et comme elles passent et operent vite, trois verres seuls que j'en ai bûs, m’ont extremement soulagé.

Les logements l'année qui vienent (sic) seront pres et suffisans pour tous ceux qui viendront. On pourra meme loger dans le beau bourg de Morjex et envoier querir les eaux dans des bouteilles. Le voiage du porteur se fera en demi heure. Madame la comtesse d'Osase $^{61}$ est resolue de loger, quand elle reviendra, à Morjex où il y a de belles maisons; dans celle du $\mathrm{S}^{\mathrm{r}}$. Tillier ${ }^{62}$ nostre secretaire, il y a 25 . belles chambres. Il y en a d'autres aussi belles.

(58) Sur les inondations qui eurent lieu en 1680, cfr. Mgr J.-M. Duc, Histoire de l'Eglise d'Aoste, éd. cit., p. 365 .

(59) «Terme très répandu dans la Suisse Française: Dégât causé par la gelée, la grêle, une ravine, un éboulement» (Littré).

(60) Il s'agit de Brosso, localité sise sur la crête qui sépare la Valchiusella de celle de la Doire, en amont d'Ivrée. Les sources de la Valchiusella sont nombreuses. Cfr.: S. BALP, Le sorgenti minerali della provincia di Torino, éd. cit., pp. 13-16.

(61) La francisation de ce toponyme rend son identification sujette à caution. Il pourrait s'agir, en effet, d'Osasco, ou plutôt, d'Osasio, ou Osazio.

S'il faut lire Osasco, il s'agit probablement de la comtesse Giulia Maria Caterina Malabaila di Cantarana épouse, depuis 1670, du comte Carlo Amedeo Cacherano di Osasco. Cfr. A. Manno, Patriziato subalpino, t. III, p. 51. Il nous reste deux lettres de cette comtesse dans la série Lettere particolari, liasse 12 de la lettre $O$ et trois dans la liasse 13 de la même lettre. Dans la première du 14 mai 1682 (liasse 12) à la duchesse Marie-Jeanne-Baptiste, elle se plaint de la mort de son mari, tandis que dans la dernière, du 3 mai 1686, adressée au premier secrétaire Charles-Joseph-Victor de
Saint-Thomas, elle se dit souffrante et demande la permission de se retirer dans son château d'Osasco. Toutefois, elle fait remarquer à son correspondant que son château est occupé par des prisonniers: les «barbets», c'est-à-dire, les ministres vaudois et elle en demande la disponibilité.

Si, par contre, il faut lire Osasio, ou Osazio, dans ce cas, il s'agirait d'Angélique de Disimieux, morte à Turin le 15 novembre 1695. Elle s'était mariée, en 1658, avec le comte Alessandro Gherardo Maria Giuseppe Ingazio Scaglia di Verrua († Verceil le 13 avril 1673). Cfr. MANNo, Patriziato subalpino, t. XXIV, p. 348. Aucune lettre de cette comtesse ne semble nous être parvenue.

(62) Il s'agit de Eugène-Gaspard de Tillier, né à Morgex en 1630, oncle paternel du plus connu Jean-Baptiste, lui aussi secrétaire du duché d'Aoste et historien illustre de sa patrie, mort en 1744. Cfr. G. PIgnet, Eugenio Gaspare De Tillier uomo d'arme $e$ di legge (1630-1699), in La Valle d'Aosta. Relazioni e comunicazioni presentate al XXXI Congresso Storico Subalpino di Aosta, 9-10-11 settembre 1956. Sotto gli auspici della Amministrazione Autonoma della Valle d'Aosta, 1958-1959, en 2 voll. t, II, pp. 693-727. Eugène-Gaspard fur nommé secrétaire du duché d'Âoste, en 1668, grâce à l'intervention 
(Ecrit à l'envers en baut duf. 2v.)

Si V.A.R. desiroit se divertir agreablelement, elle n'auroit qu'à faire l'honeur à l'entrepreneur qui va à Thurin de lui doner audience. Ses gestes, son langage, sa mine sont des originaux.

Ibid., pièce 137 non datée $[1687 \text { ? }]^{63}$.

Madame,

je ne veux plus me pleindre, ni me justifier. Je mets en oeuvre le conseil de S. François de Sales, qui console les mal heureux, en leur disant, qu'ils peuvent une fois se defendre et aprés laisser à Dieu le soin de leur pleine justification. Il le prend, Madame, ce soin notre adorable et juste Maitre et pour une premiere et authentique marque de la protection qu'il daigne rendre à mon innocence, il verse toute l'abondance de ses benedictions à la source precieuse de Cormaieul laquelle, meme de tout temps, a eu le nom de fonteine Victoire, pour montrer que ses eaux divines triomphaient des calomnies des meschans. Je prens la liberté, Madame, de vous envoier les atestations ${ }^{64}$ de $\mathrm{M}^{\mathrm{r}}$. le Marquis de Cane ${ }^{65}$ et de M. le medecin Ravet ${ }^{66}$. Elles justifient demonstrativement la vertu des nos eaux et serviront d'un monument eter[n]el(?) à la pieté de V.A.R. qui les a soutenues et qui a plus merité, par cette protection utile à tout le monde, que si elle avoit bati et doté cent monasteres. Au nom de Dieu, Madame, ne croiés plus ces ignorans imposteurs. Tenez vous, s'il vous plaist, à vos vieux et sûrs serviteurs. J'aimerois mieux estre mis en mille pieces que de vous tromper. Aussi suis-je plus que tous les homes du monde et avec mes infinis respects, Madame, de V.A.R. tres humble et tres obeissant sujet et serviteur,

D. Albert E. D'Aoste.

\section{XVI}

Ibid., pièce 121, un bifeuillet non daté, mais autographe. Au verso du f. 2 verso une autre main a écrit: «L'Evesque d'Aouste. 1680».

Madame,

je n'ai point hesité à prendre la liberté d'envoier à V.A.R. la lettre cy jointe ${ }^{67}$ par l'assurance que j'ai qu'elle vous sera agreable et c'est toute ma passion de lui ecrire des choses qui aient le bon-heur de lui plaire. Je la supplie tres humblement d'ordoner à $\mathrm{M}^{\mathrm{r}}$. le president Palavisinn ${ }^{68}$ de comettre ici à quelqu'un le soin de visiter les digues que

de son frère le chanoine Jean-Claude et de Mgr. Bailly (Ibid., pp. 707-08). Sur Jean-Baptiste, cfr. A. FRUTAZ, Le fonti per la storia della Valle d'Aosta, Roma, Edizioni di Storia e Letteratura, 1966 («Thesaurum Ecclesiarum Italiae», I, 1), pp. 20-22. Ce dernier est l'auteur, entre autres, du Nobiliaire $d u$ duché D'Aoste, édité par les soins d'André Zanotto, Aoste, Editions de la Tourneuve, 1970, où il est question d'Eugène-Garpard (pp. 596-98), de son frère Jean-Michel (p. 598) et du fils de ce dernier Jean-Baptiste (p. 599).

(63) Puisque Mgr Bailly fait état d'une «attestation» du médecin Ravetti, cette lettre pourrait remonter à 1687, la mission de Ravetti et du chimiste
Campeggio ayant eu lieu cette année-là. Cfr. la note 50 précédente, document XI.

(64) Ces deux «attestations» ne semblent pas avoir survécu.

(65) Cfr. la pièce transcrite sous le numéro XIII et le billet joint à la lettre suivante. Le graphie de ce nom est douteuse ici.

(66) Pour Gaspare Antonio Ravetti, cfr., G.G. BonIno, Biografia medica piemontese, op.cit., pp. 439-40.

(67) Il pourrait s'agir de la pièce 122 reproduite ici sous le numéro XVII.

(68) Cfr. la note 57 précédente. 
l'entrepreneur Canove a faites pour mettre la fonteine Marie à couvert des inondations de la riviere et de paier ce qui reste des $2500 £ £$ qu'il a promises à cet architecte, la besogne faite. Elle est finie et le bon home y perd assurement. V.A.R. aura, s'il lui plaist, la bonté de l'indemniser; une dizaine de pistolles pourront le contenter.

Ceux qui voient ces machines et particulierement le beau pont, le plus beau de la province, que cet habile maistre a fait, les admirent. Enfin, Madame, l'eau de votre fonteine a plus d'efficace que celle des autres. Il a fallu y revenir, parce qu'elle est plus temperée par l'abondance de son acier $^{69}$ et les autres sont plus fortes par l'abondance de vitriol.

Dieu est admirable dans toutes ses euvres. Il a mis plus de vitriol dans les deux nouvelles fonteines afin qu'on put les transporter et les faire boire à ceux qui ne peuvent pas venir les boire sur les lieux, car elles perdent en chemin quelque peu de leur force et il en a mis moins dans votre fonteine pour rendre son eau plus temperée et plus utile aux persones qui la prenent dans sa source. Toute la terre qui en peut proffiter sera obligée à V.A.R. de cet avantage et moi je serei eternellement, comme je le dois, Madame, de V.A.R. tres humble et tres obeissant sujet et serviteur,

D. Albert E. D'Aoste.

\section{XVII}

Ibid., pièce 122, non datée. Au verso du f. 2, une autre main a écrit «1680. L'Evesque d'Aouste». Il s'agit d'un bifeuillet écrit sur trois pages plus une feuille encartée écrite seulement sur le recto.

\section{Madame,}

il est sans doute que votre fonteine est triumphante; prenez s'il vous plaist la peine de lire la copie d'un article de la derniere lettre que j'ai receue de M. le Marquis de Canei (?) et que j'ai renfermée dans celle-ci et V.A.R. y verra une preuve incontestable de la victorieuse fontaine Marie. Ce Marquis a infiniment d'esprit et a fait dix ans durant une exacte experience des eaux de S. Maurice ${ }^{70}$ et puis qu'il a trouvé les vostres, Madame, plus excellentes que les autres, il faut le croire.

V.A.R. pourra, s'il lui plaist, sur cette grande autorité commander à M.le medecin Torini ${ }^{71}$, d'envoier partout son manifeste ${ }^{72}$ pour rendre publique la vertu de la

(69) La lecture est indubitable. Notre évêque voulait-il dire que l'eau de cette source était «acidulée»? Cfr. B. BERTINI, Idrologia minerale, ossia descrizione di tutte le sorgenti d'acque minerali note sinora negli Stati di S. M. il re di Sardegna, éd. cit., pp. 147-53, Divisione d'Aosta. Capo unico, provincia d'Aosta, $\$ 1$, Delle acque acidole di Courmayeur, où il décrit celles de La Victoire et de La Marguerite.

(70) La source de Saint-Maurice, à laquelle fait allusion Mgr Bailly, est vraisemblablement celle de Borgofranco, une localité sise à six kilomètres en amont d'Ivrée. Cfr. S. BALP, Le sorgenti minerali della provincia di Torino, éd. cit., pp. 10-13.

(71) Giovanni Bartolomeo Torrini (Lentosca, 12 juillet 1635 - 13 juillet 1708) était le fils de Giulio. Sa famille était originaire de Lantosque, actuellement chef-lieu du département des Alpes-Maritimes dans l'arrondissement de Nice. Giovanni Bartolomeo a été premier médecin de S.A.R. et protomédecin général des États de Savoie. Cfr. G.G. Bonino, Biografia medica piemontese, éd. cit, pp. 397-400, notice 1657. A. Manno, Patriziato su- balpino, op. cit., t. XXVI, p. 244. AST, Lettere particolari, liasse 19 de la lettre T, huit lettres qui s'échelonnent de 1662 à 1703, dont aucune ne concerne les sources de Courmayeur. Le 16 janvier 1664, Giovanni Bartolomeo Torrini exécuta l'autopsie sur Françoise d'Orléans, première femme de Charles-Emmanuel II. Cfr. S. Vignali, Le bref séjour de la «Colombina d'amore» à Turin. Relation du médecin Bartolomeo Torrini sur les causes de son décès, «Studi Francesi», XLIX, 1, 2005, pp. 97-107. A. BARGONI, Considerazioni medico-cliniche, Ibid., pp. 108-114. Son père, Giulio Torrini, a été lui aussi protomédecin et bibliothécaire des ducs de Savoie. Il a rédigé un catalogue de la bibliothèque ducale en 1659. Cfr., à ce propos, le mémoire de maîtrise présenté, sous la direction du Professeur Marziano Guglielminetti, à la Faculté des Lettres et Philosophie de l'Université de Turin, durant l'année académique 1990-1991, par Mauro AlBENGA, Inventario della Biblioteca ducale del protomedico e bibliotecario Giulio Torrini (1659).

(72) Pour son ouvrage intitulé: Parere intorno 
fonteine Marie et, ouvrant le chemin qui mene à la precieuse source de tous biens, Marie, fermer celui qui conduit à $\mathrm{S}^{\mathrm{t}}$. Maurice. Et certes, Madame, il ne faut pas avoir de grandes lumieres pour tomber d'acord que Marie est plus favorable et de plus facile à boire que Saint Maurice.

J'ay une seconde remontrance à faire à V.A.R., c'est, Madame, que vostre fonteine ajoute de nouveaux embelissemens aux belles personnes, et aux laides de nouvelles laideurs et ainsi V.A.R. aura la bonté de faire publier ces divers effets dans vostre cour et à Thurin afin que les dames en proffitent toutes positivement, ou negativement. Que cela est fin, Madame, n'en etes vous pas d'acord? Nous allons voir tout le sexe beau et difforme accourir à vostre fonteine, car il n'est pas une femme qui veuille passer pour laide, quelque laideur qu'elle puisse etre. Au moins, Madame, nous esperons d'avoir l'honeur de voir, l'année qui vient, boire vos eaux au roy et à la reyne de Chypre ${ }^{73}$ qui doneront à ces belles liqueurs tout ce qu'elles ont ordre de doner aux persones bien faites et qui, par consequent, devront à ces deux magestés l'acroissement de leurs beautés. J'atans ce miracle impatiemment et suis plein de respect, Madame, de V.A.R. tres humble et tres obeissant sujet et serviteur,.

D. Albert Illustrissime E. d'Aoste

\section{(Feuillet encarté)}

Article d'une lettre de M. le Marquis de Canei (?) ${ }^{74}$ escrite à l'Eveque d'Aoste du 23 de juillet.

Delle acque non so più come meglio e più seriosamente discorrerne se non che non s'è detto tanto mai quanto vagliono e giovano. Et ne ho conosciuto un effetto

alla natura, e qualità delle acque di Cormaggiore nel ducato di Aosta, Torino, 1688, cfr. G.G. BonINO, Biografia medica piemontese, op. cit., pp. 400. Je n'ai pu retrouver aucun exemplaire de cette publication.

(73) Allusion au titre royal auquel les seigneurs de Savoie prétendaient, depuis 1462 , suite à la cession qui leur avait été faite par Charlotte de Lusignan $(† 1462)$ fille de Jean II roi de Chypre et de Jérusalem et femme de Louis de Savoie $(\uparrow 1465)$.

(74) Aussi dans ce cas, la lecture de ce nom fait difficulté. Mgr J.-M. Duc (Histoire de l'Eglise d'Aoste, éd. cit., p. 362), qui cite une lettre de ce marquis à la duchesse, remontant à 1680, et traitant des vertus des eaux de Courmayeur, lit «Cauly». Il s'agit probablement d'une mauvaise lecture et cela pour deux raisons. Les lettres du secrétaire JeanLouis Cauly, conservées à l'AST, Lettere particolari, liasse 50 de la lettre C, fasc. 3, s'arrêtent au 9 avril 1676. Ce secrétaire est décédé le 28 juin de cette année-là et surtout J.-L. Cauly n'a jamais été marquis. Je me suis intéressé à J.-L. Cauly dans un article intitulé: «Ce cher frère...». Une lettre indédite de Claude Favre de Vaugelas à Dom Albert Bailly (28 mai 1647), in Miscellenea di studi romanzi offerta a Giuliano Gasca Queirazza, a cura di A. CornAgliotti, L. Fontanella, M. Piccat, A. Rossebastiano, A. Vitale-Brovarone. Presentazione di M. Pfister, Alessandria, Edizioni dell'Orso, 1988, en 2 vol, t. II, pp. 676-93.

Dans la lettre du 17 juillet 1680 (pièce XIII), il me semble pouvoir lire ce patronyme «Canei», qui est la forme dialectale de «Canelli» (cfr. G. GASCA
Queirazza, C. Marcato, G. B. Pellegrini, G. Petracco Sicardi, A. Rossebastiano, Dizionario di toponomastica. Storia e significato dei nomi geografici italiani, Torino, UTET, 1990, p. 130). Cette ville du département d'Asti était, en effet, un marquisat qui appartint, dès le début du XVIIe siècle (24 juillet 1602), au comte de Lomello Ambrogio Antonio Crivelli (cfr. F. GuASCO, Dizionario feudale degli antichi Stati sardi e della Lombardia (dall'epoca carolingia ai nostri tempi), Pinerolo, Tipografia già Chiantore-Mascarell.i, 1911, en 5 voll. [«Biblioteca della Società Storica Subalpina», LIV-LXVIII], t. I, p. 378). A partir du 4 juillet 1671, et encore en 1673, le marquis de Canelli était Carl'Antonio Crivelli Scarampi (cfr. AST, Sezioni riunite, Indice dei feudi, vol. 16, f. 127v. et f. 128v, à la date du 2 juin, litige entre le Patrimonial Général et le comte Giuseppe Cacherano di Villafranca et le marquis Scarampi del Cairo. Les documents auxquels renvoie cet index ont été pour la plupart brûlés en 1799. Pour le litige en question, cfr. Article 749, lettre C, liasse 26, n. 800). Les Patenti controllo finanze, registre 1704 in 1705 , ff. 179 et 185 nous permettent de savoir que ce marquisat avait été inféodé, au début du XVIII ${ }^{e}$ siècle, au comte Gian Girolamo Galleani di Barbaresco. Cfr. aussi A. Manno, Patriziato subalpino, éd. cit., t. XI, p. 77.

Puisque la lecture «Canei» et surtout son interprétation italienne sont sujettes à caution, je n'ai pas poursuivi outre la recherche pour identifier le nom de ce marquis, celui de sa femme et de son fils abbé. 
singolare qual'è che la Marchesa, la quale prima d'hora, per quanti rimedii le siano stati dati da medici et altri, non ha mai potuto purgarsi a bastanza, di presente, dette acque la purgano benignamente et abondantemente, cosa che non s'è ne anche ottenuta dall'acque di San Mauricio. Et così io le stimo molto più di quelle, per sentirne più giovamento et beneficio, sí nella detta Marchesa che nel $\mathrm{S}^{\mathrm{r}}$. Abbate e in me, et chi dirà il contrario in approvatione di queste acque non può esser che qualche appassionato, et coetera

Meum et tuum, sono le raggioni degl'appassionati.

\section{XVIII}

Ibid., pièce 93, datée, «ce 14.», sans mois ni millésime. Probablement $1687^{75}$.

Madame,

je viens d'aprendre par l'un de mes gens que j'avois doné aux sieurs Ravet et Campege pour faire l'êpreuve de nos eaux minerales, qu'ils en sont bien contents et par une science experimentale qui est la plus sûre de toutes, car ils en ont bû et elle a operé. Ils font à present leurs epreuves chimistes et j'espere que vostre bonté, Madame, me scaura bon gré d'avoir decouvert ces tresors durant sa regence dont on va voir le grand adantage, premierement par la magnificence que vous y marquerez, embelissant cette precieuse source, et la seconde par votre statue que j'y ferei dresser à v o s d e p e n s avec quelque inscription de la façon de M. de l'Escheraine ${ }^{76}$. J'en ai écrit quelque chose de ma foible maniere à $\mathrm{M}$. d'Eremanville ${ }^{77}$ qui m'a fait une lettre qui est le perpetuel eloge de V.A.R. sur vostre double et propre caractere, Madame, de Reyne Pacifique qui se manifeste dans le repos de vos peuples et dans les soins que V.A.R. daigne prendre de pacifier l'Eglise de la Val d'Aoste ${ }^{78}$. Je me flate, Madame, d'avoir plù à V.A.R. en me sacrifiant à cette paix de la façon que je me suis doné l'honeur de vous ecrire et à Monsieur nôtre digne gouverneur ${ }^{79}$.

(75) Cfr. la note 50, document XI.

(76) Jean-François premier marquis de Leschereine (6 juin 1682), mort le 30 août 1702. Il a été conseiller d'Etat, président des finances à la Chambre des Comptes (24 juin 1649), troisième président du Sénat (28 décembre 1650) et premier président de la Chambre des Comptes depuis le 5 novembre 1680. G. Galli DElla LOGGIA, Cariche del Piemonte e paesi uniti, colla serie delle persone che le banno occupate ed altre notizie di nuda bistoria, dalla fine del secolo decimo sino al dicembre 1798 , con qualche aggiunta anche del tempo posteriore, éd. cit., t. I, pp. 122 et 211. E.-A. DE ForAS, Armorial et nobiliaire de l'ancien duché de Savoie, op. cit., t. III, p. 255.

(77) Personnage non identifié.

(78) Allusion possible aux querelles qui opposaient les deux chapitres d'Aoste, celui de la cathédrale et celui de Saint-Ours, querelle qui fut composée, le 7 avril 1680, par le marquis Pallavicini. Cfr. Mgr J.-M. DUC, Histoire de l'Eglise d'Aoste, op. cit., pp. 360-62. Toutefois, Mgr Bailly fait allusion ici plus vraisemblablement au différend qui l'opposa, durant de nombreuses années, au chanoine Humbert Rol, au moins depuis 1672 . Les lettres dans lesquelles Mgr Bailly parle de cette affaire sont si nombreuses, qu'on ne peut les citer toutes. Je me borne à renvoyer à celle du 11 septembre 1675 (AST, Lettere vescovi. Aosta, liasse 1 , fasc. 7 , pièce 96). Le Sénat de Savoie mit fin provisoirement à cette querelle par un arrêt du 23 janvier 1681, mais Humbert Rol continua sa dispute encore longtemps après. Mon ancienne étudiante Eliana Padello a transcrit et commenté tous les arrêts du Sénat de Savoie, dans les pp. 376-428 de son mémoire de maitrise intitulé Lettres inédites de Mgr Albert Bailly. Aoste, 1675-1684 présenté à la Faculté des Lettres de l'Université de Turin durant l'année académique 1991-1992. Ces pièces sont conservées aux Archives Départementales de Chambéry, série $B$.

(79) Thomas Foelix Ferrero Fiesque Acciaioli, marquis de La Marmora et Canosio, fut créé gouverneur par lettres patente du 24 janvier 1678 et maintint cette charge jusqu'au mois de février 1691. J.-B DE TILLIER, Historique de la Vallée d'Aoste. Première édition intégrale ornée des planches originales, Aoste, Imprimerie ITLA, 1966, p. 454. Le texte manuscrit de l'Historique a été transcrit par les chercheurs des Archives Historiques Régionales et revu par André Zanotto. L'édition a été préfacée par Mgr Aimé-Pierre Frutaz. 
Il ne reste qu'à mettre fin à cette bagatelle de signature ${ }^{80}$, qui est veritablement une bagatelle, car qu'importe à la Courone et au Vibaillif que le premier comis signe. Je le repete, il importe à l'un et à l'autre qu'il signe, bien loin de prejudicier à leurs droits, pour toutes les raisons que j'ai aportées dans mon factum...

\section{XIX}

Ibid., pièce 115, datée d'Aoste «ce 22. de $X^{\text {bre }} 1679 »$. Cette lettre n'est pas autographe et elle est incomplète.

\section{Monsieur ${ }^{81}$,}

je prie nostre Sauveur Jesus Christ de verser à V.E. une plenitude de graces dans le retour de la solemnité de sa nativité. Il nous respand ici des benedictions pour le corps, aiant trop de bonté pour restreindre ses faveurs infinies seulement aux spirituelles. Je prens la liberté, Monsieur, de vous envoier deux attestations ${ }^{82}$ qui confirmeront cette verité. Deux manieres d'hidropiques fûrent (sic) inspirés par Aiglés ${ }^{83}$, la premiere et la plus belle nymphe des eaux, de boire celles de nostre miraculeuse fontaine et elle les a gueris. Je l'appelle miraculeuse par rendre si tost la santé aux malades dans la plus grande rigueur de l'hyver. Je vous supplie trés humblement, Monsieur, de regaler M.R., qui protege tant cette fontaine, de cette relation. Si j'estois capable de lui souhaiter une legere debilité, comme je lui desire une santé aussi parfaite qu'elle, ce seroit seulement qu'elle lui vint pour l'obliger à recevoir ici de la fontaine, qu'on appellera desormais la fontaine Marie, pour reconoissance des soins qu'elle prend de la proteger, une entiere guerison ........

(80) Aussi cette «bagatelle» avec le vibailli trâ̂nait depuis 1668, cfr. Mgr J.-M. Duc, Histoire de l'Eglise d'Aoste, pp. 355-57. A ce moment-là, le vibailli était Victor-Amé Carron de La Tour, le propre frère du premier secrétaire de l'administration savoyarde Guillaume-François Carron de SaintThomas. Il exerça cette charge du 20 mai 1656 jusqu'en 1673. Ses successeurs furent Bernardin de Malherbe de 1673 à 1675 et Blaise Beltram de 1675 à 1692. Cfr. J.-B DE TilLier, Historique de la Vallée d'Aoste, éd. cit., pp. 468-69.

(81) Le destinataire est Charles-Joseph-Victor Carron de Saint-Thomas, fils aîné et successeur de Guillaume-François dans la charge de premier secrétaire de l'administration du duché, après la mort de ce dernier survenue le 30 novembre 1677. Cfr. A. Manno, Il patriziato subalpino. Notizie di fatto, storiche, genealogiche, feudali ed araldiche desunte da documenti, Firenze, Stabilimento Giuseppe Civelli, 1895-1906, 2 voll. imprimés et vingt-neuf dactylographiés déposés à la Biblioteca Nazionale e Universitaria, à la Biblioteca Reale de Turin et à l'AST, t. IV, p. 103-05.

(82) Ces «attestations» ne semblent pas avoir été conservées.

(83) Églé est le nom de la très belle naïade qui, dans la sixième églogue de Virgile, réveille Silène endormi et l'oblige à chanter un poème. 